\title{
ULTRASTRUCTURAL PATHOLOGY OF RECTUM AND SKIN BIOPSY SPECIMENS IN LYSOSOMAL STORAGE DISEASES
}

\author{
M. TANIIKE ${ }^{1}$, T. YAMANO ${ }^{2}$, M. SHIMADA ${ }^{2}$, K. INUI $^{1 *}$ \\ AND S. OKADA ${ }^{1}$
Department of Pediatrics ${ }^{1}$, Osaka University Hospital, Osaka 553 and Department of Pediatrics ${ }^{2}$, Shiga University of Medical Science, Otsu 520-21

\begin{abstract}
Ultrastructural examinations of skin and/or rectal biopsy specimens were performed in 45 cases of various lysosomal storage diseases, including Pompe's disease, mucopolysaccharidoses I-IV, GM \& GM $\mathbf{G M}_{2}$ gangliosidoses, Niemann-Pick disease, Gaucher's disease, metachromatic leukodystrophy, Fabry's disease, sialidosis, I-cell disease, mucolipidosis III, mannosidosis, fucosidosis, and ceroidlipofuscinosis. We found characteristic storage inclusions in all disorders except for Gaucher's disease, probably indicating the absence of storage outside the reticuloendothelial system. In some cases of adult-onset neurolipidosis and ceroid-lipofuscinosis, the examination of the rectal submucous plexus neurons was important to detect characteristic inclusions, suggesting some superiority of the rectal biopsy to the skin biopsy in these conditions. On the contrary, because of the absence of the myelinated fibers in the rectal mucosa, the skin biopsy seems to be superior to the rectal one in diseases affecting the myelin sheath primarily. These morphological studies about skin and/or rectal biopsy specimens are useful to support the diagnosis obtained by the biochemical methods, to be a clue to diagnosis of cases in which biochemical defects of the disease are still unclear, and to make a probable diagnosis of some lysosomal storage diseases in atypical cases showing neurodegeneration.
\end{abstract}

Lysosomal storage diseases are a group of disorders in which a deficiency of a lysosomal enzyme leads to the progressive intralysosomal storage of unhydrolyzed substrates. In the past, the diagnoses of the diseases were mainly established by the morphological findings (6), and the stored substrates and the enzymatic defects have been clarified with the advances of biochemical studies (9). But recent studies about atypical cases of lysosomal storage diseases have shown that the "one enzyme deficiency-one disease" theory is not always the case. For example, the variant cases of $\mathrm{GM}_{2}$ gangliosidosis (variant AB), metachromatic leukodystrophy and Gaucher disease, in which the enzyme proteins are not deficient, are the results of the deficiency of the sphingolipid activator proteins necessary for enzymatic hydrolysis of the substrates (24 ), and the deficiency of the protective protein necessary for stabilization of sialidase and $\beta$-galactosidase leads to a combined deficiency of both enzymes (9). With these advances in understanding the etiology of lysosomal storage diseases, the morphological examinations have naturally changed from merely diagnostic methods to tools for studying pathogenesis of these diseases.

\footnotetext{
* To whom all correspondences should be addressed.
} 
Lysosomal storage diseases are now classified into glycogenosis, mucopolysaccharidoses, lipidoses, mucolipidoses and others according to the stored substrates. Based on our experiences obtained by the morphological studies of skin and/or rectal biopsy, we here describe the ultrastructural characteristics of the lysosomal inclusions in neurons, histiocytes, axons, Schwann cells, endothelial cells, and fibroblasts in these lysosomal storage diseases from a mainly diagnostic point of view in order to evaluate the usefulness and superiority of such examinations in various conditions. The possible role of morphology in the future is also briefly discussed.

\section{MATERIALS AND METHODS}

Of 147 patients diagnosed to be affected with various lysosomal storage diseases in our laboratory for 30 years, 45 patients were subjected to this morphological study. The diagnoses were confirmed by biochemical or morphological results (when the biochemical defect is still unknown) in addition to the clinical features. The deficient enzymes (or proteins) and the main stored substrates in these diseases are summarized in Table 1.

Biopsies were taken from the skin and/or the rectum in each patient with informed consent. Skin biopsy specimens were taken aseptically from the forearm by use of a sterile scalpel blade (8). The result was exceptionally mentioned about conjunctival biopsy specimens from two siblings with mannosidosis because neither skin nor rectal biopsy of patients with this disorder were undertaken in our laboratory (13).

The rectal mucosa specimens were taken using Watson's intestinal biopsy capsule from the rectal mucosa about $5 \mathrm{~cm}$ inside the anus (14). The specimens thus obtained were divided into small blocks suitable for electron microscopic studies. They were fixed in $5 \%$ phosphate-buffered glutalaldehyde solution for $2 \mathrm{hr}$-overnight, washed in the buffer, and postfixed in $1 \%$ osmium tetroxide solution for $2 \mathrm{hr}$ at $4^{\circ} \mathrm{C}$. After dehydration in graded solutions of ethyl alcohols, blocks were embedded in epoxy resin. Ultrathin sections stained with uranyl acetate and lead citrate were examined on an electron microscope.

In some cases where the examination of submucous plexus in the rectum was necessary, the rectal specimens were sliced 50-100 $\mu \mathrm{m}$ thick with an Oxford vibratome for easy cell orientation.

\section{RESULTS}

The skin biopsy specimens consisted of the epidermis and dermis with or without subcutaneous fat tissue. The rectal tissues included the mucous membrane, the lamina propria and muscularis mucosae with or without submucous connective tissues. Various abnormalities were found mainly in myelinated and unmyelinated fibers, Schwann cells, endothelial cells, skin fibroblasts in the dermis of skin biopsy specimens, and unmyelinated fibers, Schwann cells, endothelial cells, fibroblasts, histiocytes and neurons in the submucous plexus in the rectal submucous connective tissues.

The results are summarized in the Table 2 (15).

Glycogen Storage Disease II, Juvenile type

Examination of the skin biopsy specimen revealed the clustered glycogen 
TABLE 1. Biochemical characteristics of the diseases under study

\begin{tabular}{|c|c|c|}
\hline Disease & Deficient Enzyme (Protein) & Main Stored Substance \\
\hline \multicolumn{3}{|l|}{ Glycogenosis $I I$} \\
\hline juvenile & $\alpha$-Glucosidase & Glycogen \\
\hline \multicolumn{3}{|l|}{ Mucopolysaccharidoses } \\
\hline IH & $\alpha$-Iduronidase & \\
\hline IS & $\alpha$-Iduronidase & \\
\hline II & Idurinate sulfatase & Mucopolysaccharides \\
\hline IIIB & $\alpha$-N-Acetyl-Glucosaminidase & \\
\hline IVA & Galactose 6-sulfatase & \\
\hline \multicolumn{3}{|l|}{ Lipidoses } \\
\hline \multicolumn{3}{|l|}{$\mathrm{GM}_{1}$ gangliosidosis } \\
\hline \multicolumn{3}{|l|}{ infantile } \\
\hline juvenile & $\beta$-Galactosidase & $\mathrm{GM}_{1}$ ganglioside \\
\hline \multicolumn{3}{|l|}{ adult } \\
\hline \multicolumn{3}{|l|}{$\mathbf{G M}_{2}$ gangliosidosis } \\
\hline \multicolumn{3}{|l|}{ Sandhoff } \\
\hline infantile & $\beta$-Hexosaminidase & $\mathrm{GM}_{2}$ ganglioside \\
\hline adult & $A \& B$ & \\
\hline \multicolumn{3}{|l|}{ Tay-Sachs } \\
\hline infantile & $\beta$-Hexosaminidase $A$ & \\
\hline \multicolumn{3}{|l|}{ Niemann-Pick disease } \\
\hline type A & Sphingomyelinase & Sphingomyelin \\
\hline type C & Unknown & (\& Cholesterol) \\
\hline \multicolumn{3}{|l|}{ Gaucher's disease } \\
\hline \multicolumn{3}{|l|}{ II } \\
\hline III & $\beta$-Glucosidase & Glucocerebroside \\
\hline \multicolumn{3}{|l|}{ Metachromatic } \\
\hline leukodystrophy & Arylsulfatase A & Sulfatide \\
\hline \multicolumn{3}{|l|}{ late-infantile } \\
\hline Fabry's disease & $\alpha$-Glucosidase & Globotriaosylceramide \\
\hline \multicolumn{3}{|l|}{ Mucolipidoses } \\
\hline Sialidosis & Sialidase & \\
\hline \multirow[t]{2}{*}{ Galactosialidosis } & $\beta$-Galactosidase $\&$ Sialidase & \\
\hline & (Protective protein) & \\
\hline I-cell disease & Phosphotransferase & Glycoproteins \\
\hline Mucolipidosis III & Phosphotransferase & \\
\hline Mannosidosis & $\alpha$-Mannosidase & \\
\hline Fucosidosis & $\alpha$-Fucosidase & \\
\hline \multicolumn{3}{|l|}{ Others } \\
\hline $\begin{array}{l}\text { Ceroid-lipofuscinosis } \\
\text { juvenile } \\
\text { late-infantile }\end{array}$ & Unknown & Unknown \\
\hline
\end{tabular}


TABLE 2. Ultrastructural characteristics of the stored material in the specimen obtained by skin and/or rectal biopsy.

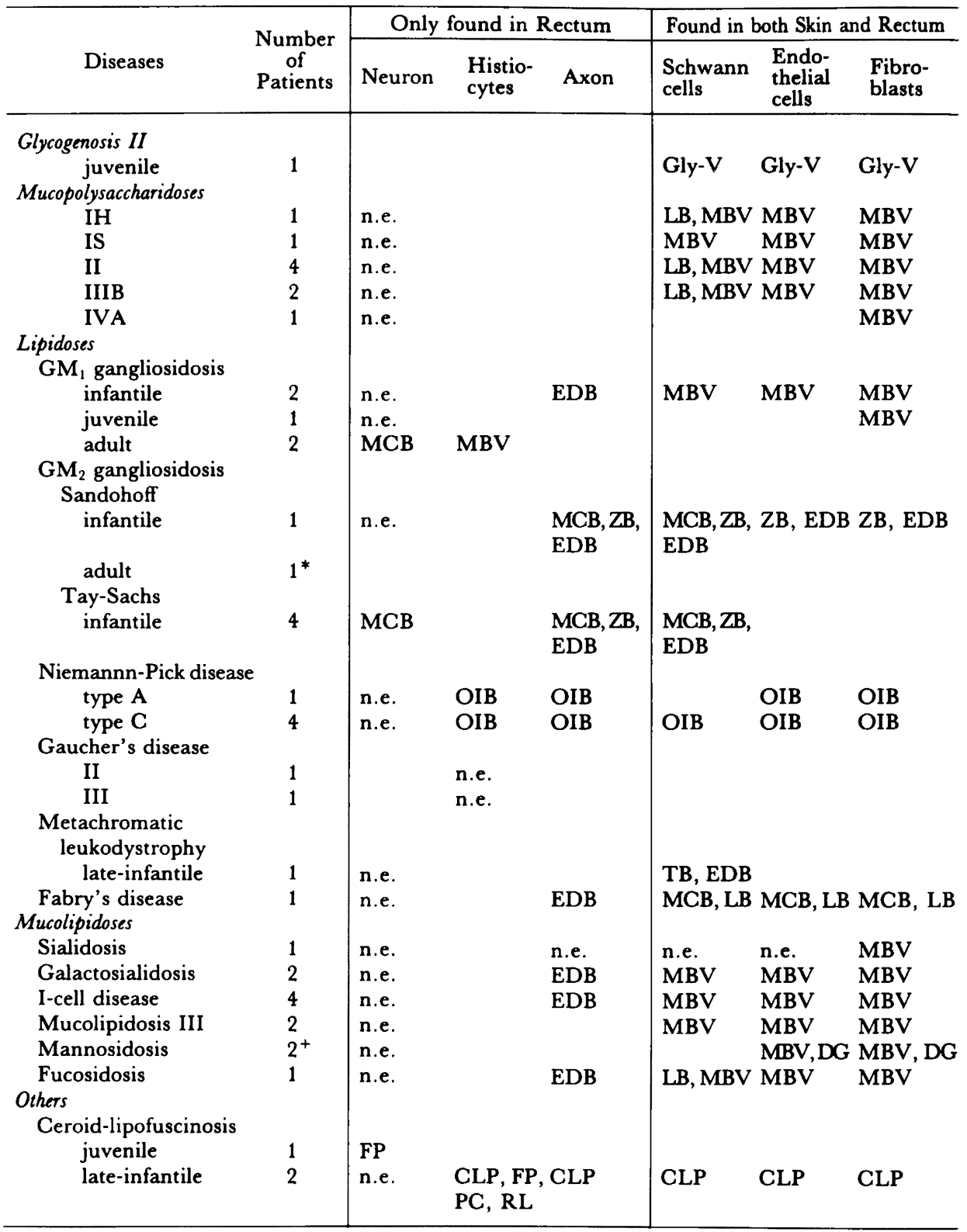

n.e: not examined, gly-V: vesicle filled with glycogen particles, MBV: membrane-bound vacuole, LB: lamellar body, MCB: membranous cytoplasmic body, ZB: zebra body, EDB: electron dense body, OIB: osmiophilic inclusion body, TB: tuffstone body, DG: dense globule, FP: fingerprint figure, CLP: curvilinear profile, PC: paracristalline figure, RL: rectilinear profile.

*: The immunohistochemical study only was performed using anti-GM $\mathrm{G}_{2}$ antibody.

${ }^{+}$: The specimens were obtained by the conjunctival biopsy. 

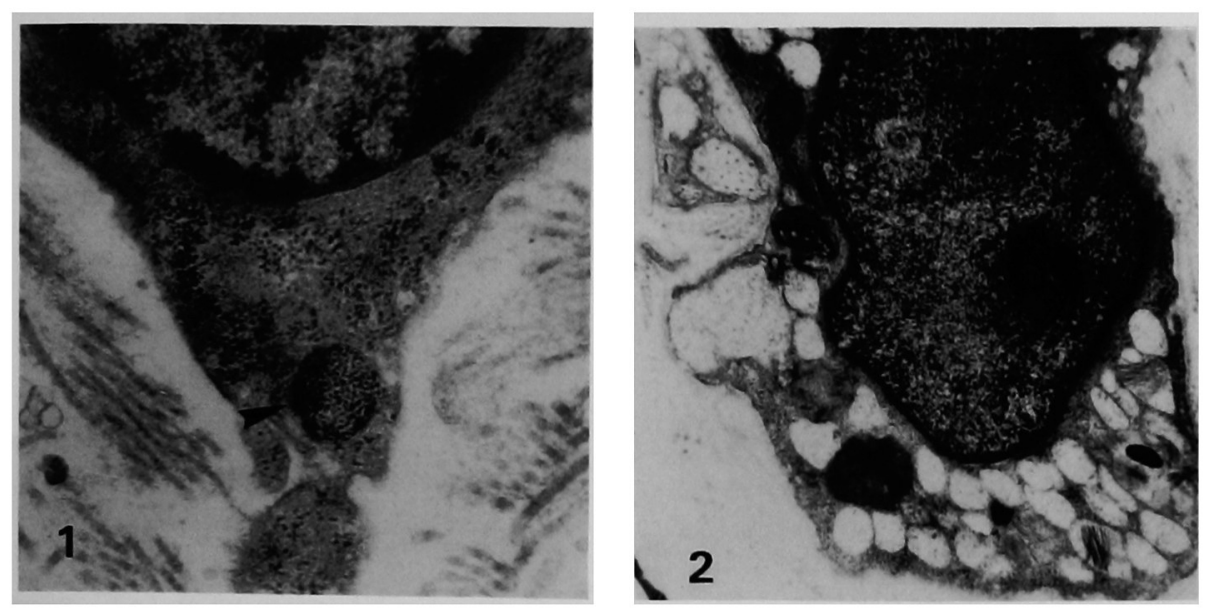

Fic. 1. Glycogenosis II, juvenile type. Dermal fibroblasts containing a membrane-bound glycogen-filled lysosome (arrowhead). $\times 20,000$

FIG. 2. Mucopolysaccharidosis IIIB. Dermal fibroblasts containing many membrane-bound vacuoles. Some vacuoles have reticulo-granular appearance. $\times 12,000$

granules enclosed within a limiting membrane in skin fibroblasts, Schwann cells, and endothelial cells (Fig. 1). These findings are thought to be pathognomic for the disease.

Mucopolysaccharidoses (MPS) I-IV

Numerous MBV's* were noted in fibroblasts, endothelial cells, and Schwann cells (Fig. 2). Occasionally Schwann cells had lamellar bodies. Although the vacuoles of each subtype did not display any significant differences in fine structure and cell distribution, the vacuoles of MPS IVA tended to be small in number and size. Lipidoses

1) $\mathrm{GM}_{2}$ gangliosidosis

Many EDB's, ZB's and MCB's were only found in the neural tissues of the specimen with the Tay-Sachs disease (hexosaminidase A deficiency) (Fig. 3). By contrast, examinations of the tissues from a patient with Sandhoff's disease (hexosaminidase AB deficiency) revealed many MCB's, EDB's and ZB's not only in the neural tissues but also in the mesenchymal tissues such as endothelial cells and fibroblasts (Fig. 4).

2) $\mathrm{GM}_{1}$ gangliosidosis

Examination of the skin biopsy specimen of the patient with $\mathrm{GM}_{1}$ gangliosidosis, infantile type revealed the presence of MBV's in many cell types and occasionally the presence of EDB's in the axon. The number of vacuoles tended to be fewer in the juvenile type, and the fewest in the adult type. The rectal specimen of the adult onset $\mathrm{GM}_{1}$ gangliosidosis showed no abnormality except for a few MCB's in neurons of the submucosal plexus.

3) Niemann-Pick disease, type $A$ and type $C$

\footnotetext{
*: The abbreviations used in this paper are listed under the Table 2.
} 

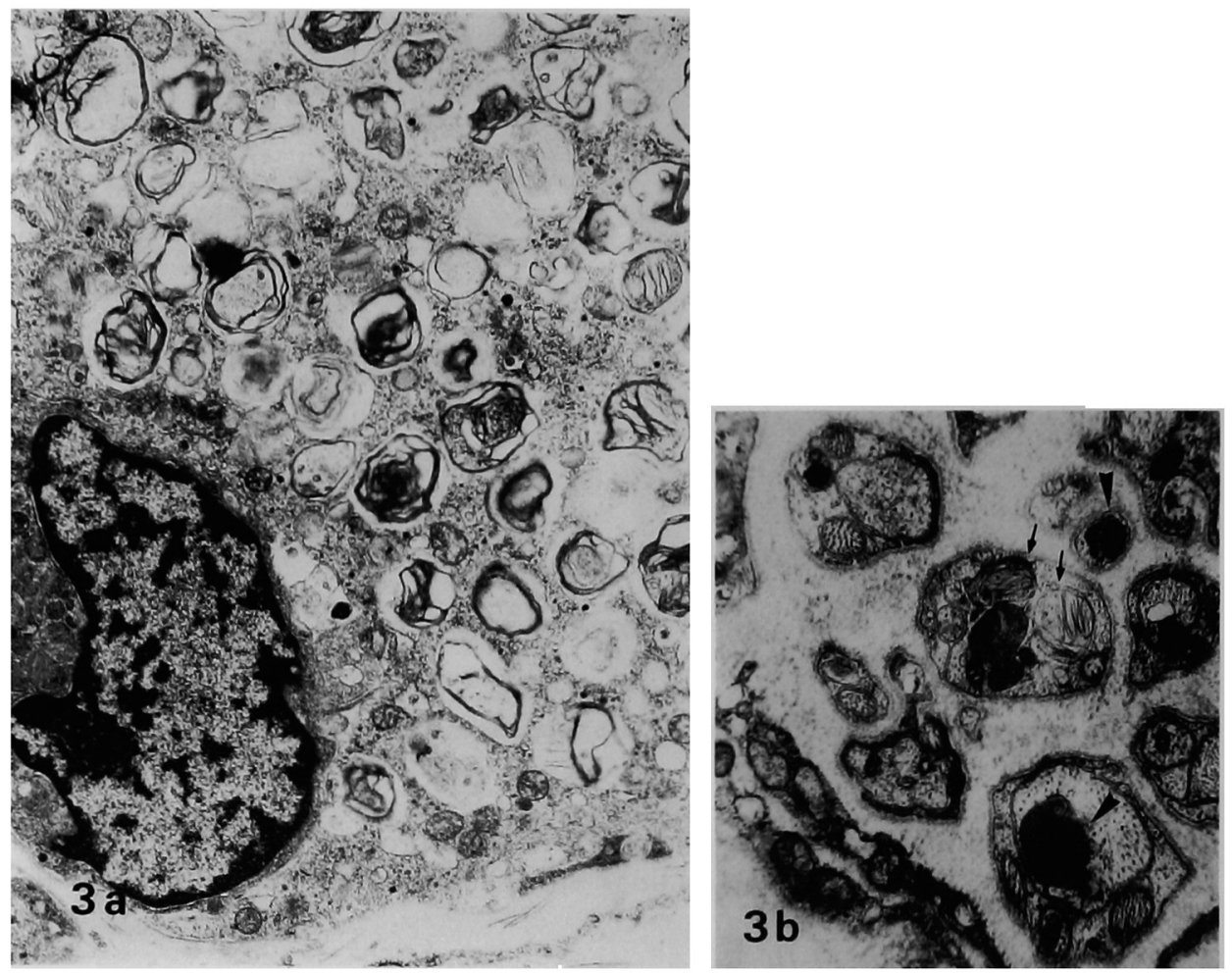

Figs. 3a, b. Tay-Sachs Disease, infantile type. a. The neuron in the submucous plexus has many MCB's. $\times 8,000$ b. In unmyelinated nerve bundles, EDB's are recognized in axons (arrowheads), whereas ZB's are recognizable in Schwann cell cytoplasms (arrows). $\times 14,000$

Many vesicles with OIB's were present in axons, Schwann cells, endothelial cells, fibroblasts and histiocytes. OIB's were different from EDB's in that they were pleomorphic and not homogeneous with lamellar or washed-out appearance. Morphological findings of type $\mathrm{A}$ and type $\mathrm{C}$ made no significant differences in skin and rectal specimens. Ultrastructural inclusions in skin and rectal biopsy specimens were somewhat different from that of "myelin-like" figures in Niemann-Pick cells in the reticuloendothelial system.

4) Gaucher diesase

No cytoplasmic inclusions were recognized in any cell types examined in the skin biopsy specimen. Rectal biopsy was not performed. Spindle or rod shaped inclusions seen in Gaucher cells in the reticuloendothelial system were not recognized in the skin biopsy specimens.

5) Metachromatic leukodystrophy

"Tuffstone" bodies (TB's) or EDB's could be observed in Schwann cell cytoplasm in skin biopsy specimens. TB was highly specific for the disease (Fig. 6).

6) Fabry's disease

MCB's, LB's and EDB's were easily observed in many cell types (Fig. 7). 


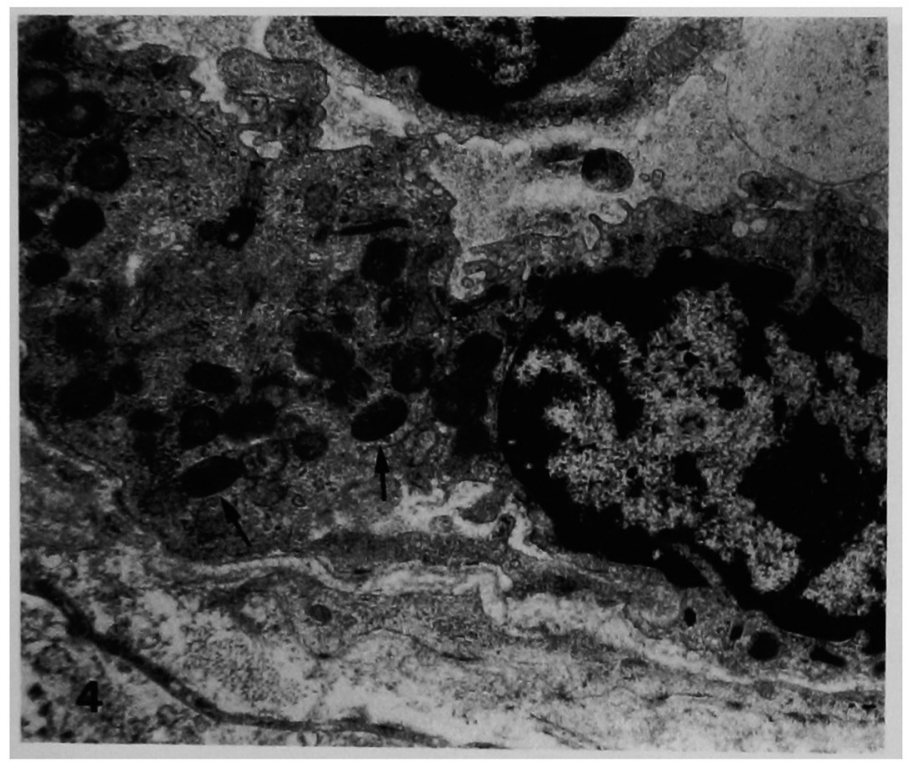

Fic. 4. Sandhoff Disease, infantile type. Vascular endothelial cells containing many MCB's (arrows). $\times 10,000$

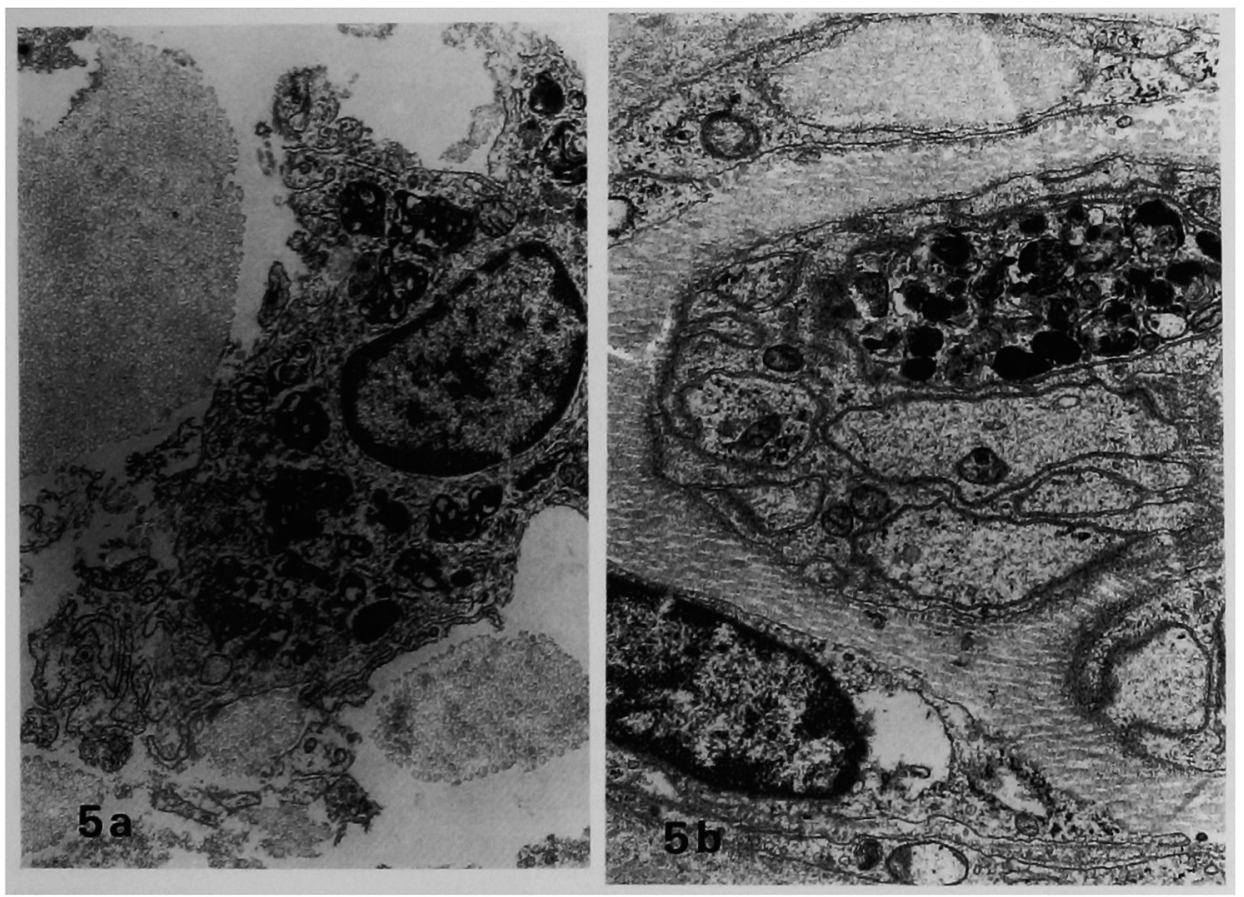

Fics. 5a, b. Niemann-Pick Disease, type C. Many pleomorphic OIB's are recognizable in a dermal fibroblast and in an unmelinated nerve fiber. a. $\times 7,800$, b. $\times 12,000$ 


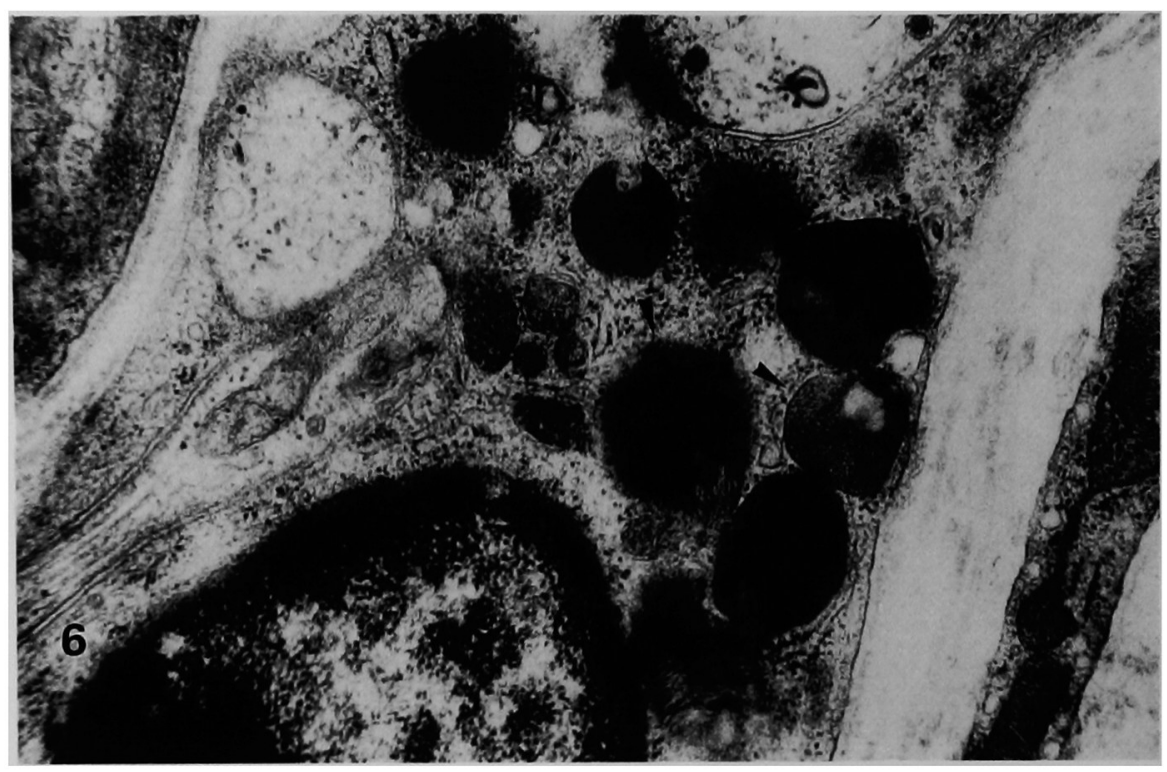

FIG. 6. Metachromatic leukodystrophy. Dermal Schwann cells have inclusions showing characteristic "tuffstone" appearance (arrowheads). $\times 20,000$

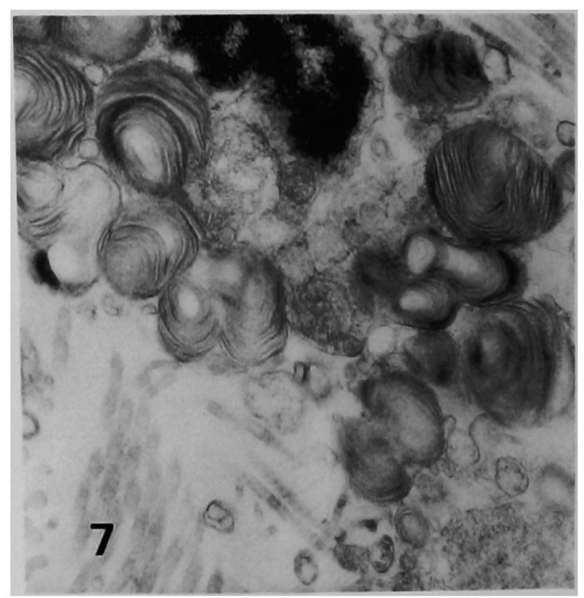

Fic. 7. Fabry's disease. Dermal fibroblasts containing many MCB's and LB's. $\times 20,000$

FIG. 8. I-cell Disease. Dermal fibroblasts containing numerous vacuoles. $\times 23,000$

FIG. 9. Galactosialidosis. An axon containing many EDB's. $\times 12,000$

FIG. 10. Mannosidosis. The dense globules in the endothelial cells. They were never recognized in other storage disorders. $\times 15,000$

FIgs. 11a, b. Ceroid-lipofuscinosis. a. Late-infantile type. Curvilinear profiles (arrowhead) are evident in the cytoplasm of Schwann cells; they are enclosed within a single membrane. $\times 9,000$ b. Juvenile type. The neuron in the rectal submucous plexus has characteristic "fingerprint" inclusions (arrowhead). $\times 38,000$ 

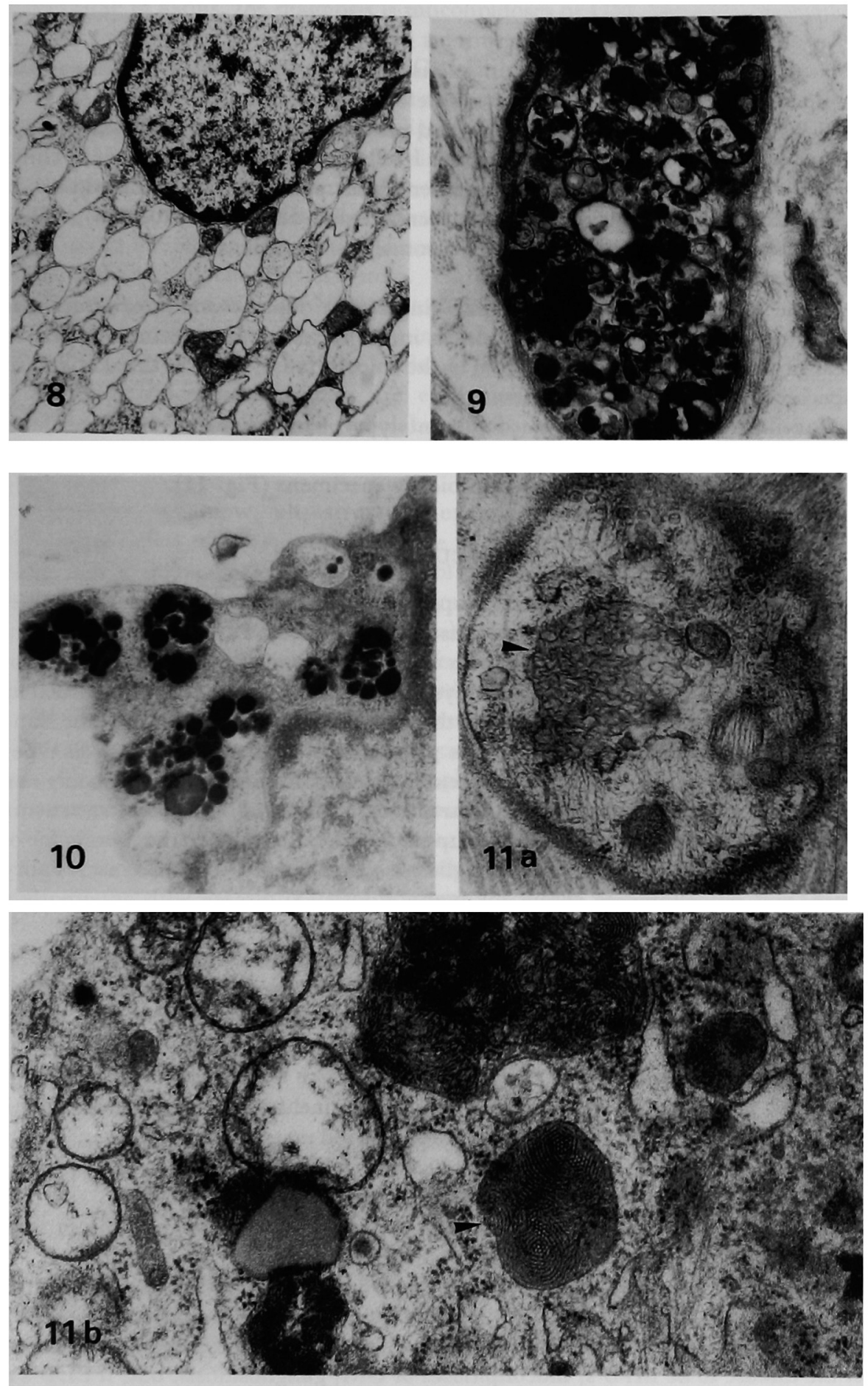


\section{Mucolipidoses}

In general , many MBV's were seen in Schwann cells, endothelial cells and fibroblasts, and EDB's were seen in axons (Figs. 8, 9). Both I-cell disease and mucolipidosis III have the same enzyme defect (phosphotransferase deficiency) and can be distinguished on clinical criteria and progression of the disease. MBV's are fewer in number in mucolipidosis III than I-cell disease in coincidence with clinical severity. The conjunctival biopsy specimens from patients with mannosidosis had many dense globules in fibroblasts and endothelial cells (Fig. 10) (13). Dense globules were not recognized in other lysosomal storage diseases.

Ceroid-Lipofuscinosis

In the specimen of the juvenile type, "fingerprint" figures were most prominently found in neurons of the submucous plexus in the rectal biopsy specimen. The rectilinear profiles were occasionally found in the smooth muscle cells in the skin and rectal specimens.

Curvilinear profiles were the dominant type of inclusions of the late-infantile type. They were found in endothelial cells, smooth muscle cells and axons in the various degree of frequency in the skin biopsy specimens (Fig. 11).

\section{DISCUSSION}

The value of the skin and rectal biopsies in lysosomal storage diseases has been proven in a very large number of cases $(1,5,6,8,12,14)$. Evaluation of topographical and ultrastructural features of inclusions indicate a disease or a group of disorders. Based upon our own results, lysosomal storage diseases can be divided into 3 groups according to the effectiveness of the morphological examination of the skin or rectal specimens: 1) diseases which have pathognomic findings: glycogenosis type 2, metachromatic leukodystrophy, each type of ceroid-lipofuscinosis, and probably mannosidosis; 2) diseases which have characteristic topography and ultrastructural features of inclusions to indicate the group of disorders, but not pathognomic for one disease: MPS's, $G_{1}$ and $\mathrm{GM}_{2}$ gangliosidoses, Niemann-Pick disease, Fabry's disease, and mucolipidoses except for mannosidosis; 3) disease with apparently no pathological findings: Gaucher disease.

In group 1, the aggregation of the glycogen particles in Pompe's disease, TB's in metachromatic leukodystrophy, and FP's or CLP's in ceroid-lipofuscinosis are highly specific and pathognomic for each disease. Although dense globules were recognized only in mannosidosis, more studies may be needed to confirm this specificity.

In group 2, morphological examinations alone are not sufficient for the definite diagnosis, since many storage diseases have inclusions morphologically indistinguishable from each other. For example, stored material in lipidoses often share common ultrastructural characteristics, because sphingolipids are polar lipids and tend to form lamellar structures. In MPS's, stored mucopolysaccharides are highly soluble in water and dissolve out during the specimen processing, resulting in empty appearances of lysosomes examined on an electron microscope. Accordingly, the tissues affected with MPS's have MBV's in common regardless of the deficient enzyme. But morphological findings are often helpful in guiding further biochemical studies. For example, MBV's in Schwann cells, endothelial cells and fibroblasts suggest the disease to be MPS's, mucolipidoses or GM $_{1}$ gangliosidosis, but, if EDB's are 
recognizable in axons, the diagnosis is mucolipidoses or $\mathrm{GM}_{1}$ gangliosidosis (Figs. 8, 9). The examination of neurons in the submucous plexus in the rectal specimens is useful to demonstrate characteristic MCB's in $\mathrm{GM}_{1}$ gangliosidosis. On the other hand, when examined cells in skin or rectal specimens have EDB's, ZB's, LB's or MCB's, the diagnosis may be one of lipidoses. Findings of pleomorphic OIB's in many cell types make a probable diagnosis of Niemann-Pick disease. The disorder in group 2 needs diagnostic biochemical methods.

In group 3, in cases of Gaucher disease, morphological examination of skin biopsy specimens offered no diagnostic information probably because of the restriction of storage in the reticuloendothelial system. Careful observation of histiocytes in the rectum biopsy specimen may show some storage.

The above results show the efficacy and the limitation at the same time of the morphological examinations in various kinds of lysosomal storage diseases.

Recently lysosomal enzymes are easily assayed by the use of synthetic 4methylumbelliferyl substrates or radiolabeled natural substrates. So, when adequate biochemical assays are available, morphological examinations may be omitted in typical cases.

However, still now, ultrastructural examinations are essential, 1) when the biochemical defect of the suspected disease is not clear, for example, Niemann-Pick disease type $\mathrm{C}$ (primary defect in cholesterol esterification?) and ceroid-lipofuscinosis, 2) when the signs and symptoms are not typical for the suspected disease: adult-type gangliosidoses, and 3) when the biochemical evidence of storage and clinical histories does not coincide with the result of the in vitro enzyme assays: activator-deficient types of $\mathrm{GM}_{2}$ gangliosidosis, Gaucher disease and metachromatic leukodystrophy (2-4).

In cases of adult-onset lipidoses and juvenile type of ceroid-lipofuscinosis, the rectal biopsy is superior to the skin biopsy because there is more chance to find storage materials in neurons of the submucous plexus in these cases (7). We recently demonstrated neuronal storage of gangliosides in adult-onset gangliosidoses using anti-ganglioside antibodies. The immunoelecton microscopical examination of the rectum of the adult-onset $\mathrm{GM}_{1}$ gangliosidosis revealed the storage of $\mathrm{GM}_{1}$ ganglioside occurs not only in MCB-containing lysosomes but also in plasma membranes (unpublished data). This finding is suggestive of the membrane dysfunction of neurons and very interesting in thinking of the pathogenesis of the diseases.

Recent advances in methodology such as immunocytochemistry and in situ hybridization will allow us to identify the stored material directly and topographically and to understand the pathogenesis and evolution of clinical symptoms $(10,11)$.

\section{REFERENCES}

1. Centerick, C. and Martin, J.J.: Diagnostic role of skin or conjunctival biopsies in neurological disorders. An Update: J. Neurol. Sci. 65; 179-191, 1984.

2. Christomanou, H., Aignesberger, A. and Linke, R. P.: Immunochemical characterization of two activator proteins stimulating enzymic sphingomyelin degradation in vitro absence of one of them in a human Gaucher disease variant. Biol. Chem. Hoppe-Seyler 367; 879-890, 1986.

3. Conzelmann, E. and Sandhoff, $\mathrm{K}$.: AB variant of infantile $\mathrm{GM}_{2}$ gangliosidosis. Deficiency of a factor necessary for stimulation of hexosaminidase A-catalyzed degradation of ganglioside $\mathrm{GM}_{2}$ and glycolipid GA . Proc. Natl. Acad. Sci. USA 75; 3979-3983, 1978. 
4. Inui, K., Emmett, M. and Wenger, D. A.: Immunological evidence for a deficiency in an activator protein for sulfatide sulfatase in a variant form of metachromatic leukodystrophy. Proc. Natl. Acad. Sci. USA 80; 3074-3077, 1983.

5. Martin, J. J. and Centerick, C.: Morphological study of skin biopsy specimen: A contribution to the diagnosis of metabolic disorders with involvement of the nervous system. $J$. Neurol. Neurosurg. Psychiatry 41; 232-248, 1978.

6. Nakai, H. and Landing, B. H.: Suggested use of rectal biopsy in the diagnosis of neuronal lipidoses. Pediatrics 20; 225-228, 1960.

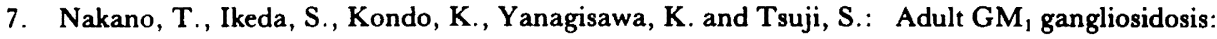
clinical patterns and rectal biopsy. Neurology 35; 875-880, 1985.

8. O'Brien, J. S., Bernett, J., Veath, L. and Paa, D.: Lysosomal storage disorders. Diagnosis by ultrastructural examination of skin biopsy specimens. Arch. Neurol. 32; 592-599, 1975.

9. Scriver, C. R., Beaudet, A. L., Sly, W. S. and Valle, D.: The Metabolic Basis of Inherited Disease, vol. II, 6th ed., McGraw-Hill, New York, 1989.

10. Taniike, M., Inui, K., Shinoda, K., Okada, S., Shiotani, Y. and Yabuuchi, H.: Correlation of subcellular localization of disease-specific inclusions and sphingolipid activator protein-1 (SAP-1) in sulfatide sulfatase-deficient fibroblasts. Acta histochem. cytochem. 21; 565-573, 1988.

11. Taniike, M., Inui, K., Hirabayashi, H., Tsukamoto, H., Nishimoto, J., Midorikawa, M., Okada, S. and Yabuuchi, H.: Immunological demonstration of $\mathrm{GM}_{2}$ ganglioside in the central nervous system of a 19-week-old fetus of Tay-Sachs disease. J. Inher. Metab. Dis. 12 (Suppl. 2); 372-374, 1989.

12. Yamano, T., Shimada, M., Okada, S., Yutaka,T., Kato, T., Yabuuchi, H. and Nakao, T.: Electron microscopic examination of skin and conjunctival biopsy specimens in neuronal storage diseases. Brain Dev. 1; 16-25, 1979.

13. Yamano, T., Shimada, M., Okada, S., Yutaka, T., Yabuuchi, H., Mitsudome, A. and Itsuki, S.: Electron microscopy of conjunctival biopsy in mannosidosis. Neurology 31; 630-632, 1981.

14. Yamano, T., Shimada, M., Okada, S., Yutaka, T., Kato, T. and Yabuuchi, H.: Ultrastructural study of biopsy specimens of rectal mucosa. Its use in neuronal storage diseases. Arch. Pathol. Lab. Med. 106; 673-677, 1982.

15. Yamano, T.: Pathology of neuronal storage disease. Its use in the supportive morphological diagnosis. Byori To Rinsho 4; 1137-1144, 1986. 